\title{
Do plano ao volume: a gramática dos planos em série como partido para a fabricação digital por méio de cortadoras a laser
}

From the plan to the volume: the grammar of plans in series as a party to digital fabrication through laser cutters

> Frederico Braida Rodrigues de Paula

Universidade Federal de Juiz de

Fora, Brasil

frederico.braida@uff.edu.br

> Fernando Lima

Universidade Federal de Juiz de

Fora, Brasil

fernando.tadeu@ufff.edu.br

> Juliane Fonseca

Universidade Federal de Juiz de

Fora, Brasil

julianearq@uol.com.br
> Vinicius Morais

Universidade Federal de Juiz de

Fora, Brasil

vinicius.morais@uff.edu.br

> Ashley Adelaide Rosa

Universidade Federal de Juiz de

Fora, Brasil

ashileya.rosa@hotmail.com

> Diogo Machado Homem

Universidade Federal de Juiz de

Fora, Brasil

diiogomachadoh@yahoo.com.br
> Izabela Ferreira Silva

Universidade Federal de Juiz de

Fora, Brasil

izabelafersil@gmail.com

> Juliana Coelho

Universidade Federal de Juiz de

Fora, Brasil

julianacoelholourenco@gmail.com

> Mariane da Paz Almeida

Universidade Federal de Juiz de

Fora, Brasil

marianedapazal@hotmail.com

\begin{abstract}
This article intent to promote discussion about the possibilities of using the plans in series reasoning as a party to digital fabrication. More specifically, this paper will address the possibilities of manufacturing objects using the logic of plans in series and laser cutting technology. To that end we assembled an identification of elements and transformations arising from the concepts of shape grammar, as well as an assessment of how to produce various objects in this sense.
\end{abstract}

Keywords: Digital Fabrication; Laser Cutting; Plans in series; Shape Grammar.

\section{Introdução}

O principal objetivo deste artigo é o de discutir as possibilidades do emprego do raciocínio dos planos em série (WONG, 2010) como partido para a concepção de objetos a serem fabricados digitalmente por máquinas de corte a laser, à luz do referencial teórico da gramática da forma (STINY e GIPS, 1972; MITCHELL, 2008). Dado que essas técnicas permitem, em essência, "a produção de componentes planos usando uma cabeça de corte que segue as instruçóes ditadas por um projeto digital para produzir elementos com formas diversas a partir de lâminas de distintos materiais" (DUNN, 2012, p.88), parte-se da premissa de que o raciocínio subjacente à concepção de formas volumétricas valendo-se dos planos em série se mostra extremamente pertinente para um amplo universo da manufatura automatizada.

Diante dos paradigmas contemporâneos de projetação e de materialização dos objetos e do ambiente construído, destacamse os equipamentos e os processos de prototipagem rápida e de fabricaçấo digital. Essas máquinas permitem tanto a produção de protótipos, modelos de testes, quanto a fabricação de produtos acabados ou elementos construtivos a serem empregados diretamente nas construçôes. De acordo com Dunn (2012, p.88), as técnicas de construção digital são, geralmente, divididas em quatro grandes categorias: (1) de corte; (2) subtrativas; (3) formativas; e (4) aditivas. Pupo (2009, p.49), por sua vez, afirma que são três os modos (ou processos) pelos quais os objetos podem ser produzidos automaticamente: (1) subtrativo, pelo desbaste do material, subdividido em (1.1) por fresas do tipo CNC e (1.2) por corte; (2) formativo, pela conformação do material; e (3) aditivo, pela sobreposição de camadas. Embora haja diferenças nessas genealogias propostas pelos autores, em termos técnicos, os processos e os equipamentos envolvidos são os mesmos.

Nesse cenário, cabe refletir sobre o raciocínio subjacente aos diferentes tipos de técnicas empregadas na manufatura automatizada, a fim de explorar amplamente a liberdade formal tecnicamente viabilizada nos dias de hoje. Neste artigo, propóe-se uma reflexão sobre as formas geradas para serem produzidas a partir de cortadoras, especificamente, as cortadoras a laser, as quais têm sido adquiridas pelas escolas de Arquitetura e Design. De acordo com Dunn (2012), o método de fabricação digital mais acessível é o corte, pois "a cortadora a laser é uma máquina que se usa cada vez mais tanto nos escritórios quanto nas escolas de arquitetura; também são usadas por maquetistas profissionais e nos laboratórios de projeto. Se trata de uma ferramenta importante pela grande quantidade de funçôes que oferece" (DUNN, 2012, p.90).

Deve-se, ainda mencionar, em termos metodológicos, que este artigo é resultado das análises críticas do processo de trabalho e dos 
objetos fabricados durante um workshop desenvolvido com alunos do ciclo fundamental do curso de Arquitetura e Urbanismo, sob a coordenação dos professores do Grupo de Pesquisa das Linguagens e Expressóes da Arquitetura, Urbanismo e Design (LEAUD), no âmbito do Laboratório de Prototipagem e Fabricação Digital, vinculado ao Departamento de Arquitetura e Urbanismo da Universidade Federal de Juiz de Fora (UFJF).

Uma gramática para os planos em série

Pode-se observar uma aproximaçáo entre a lógica empregada na operação técnica (bidimensional) das cortadoras a laser e o raciocínio da concepção da forma volumétrica a partir dos planos em série, os quais nada mais são do que um modo de pensar em seçôes transversais (de intervalos regulares) de uma volumetria (WONG, 2010, p.247). Tal aproximação se mostra viável, uma vez que "este processo de construção é talvez o que mais se pareça com os métodos convencionais de produção de maquetes e protótipos dado que os componentes são cortados a partir de lâminas de material e depois são juntados para dar forma a propostas tridimensionais." (DUNN, 2012, p.90).

No processo de geração de uma forma volumétrica a partir dos planos em série, de acordo com Wong (2010), as seçôes transversais podem ser dispostas empregando a repetiçáo exata ou a gradação (Figura 1).

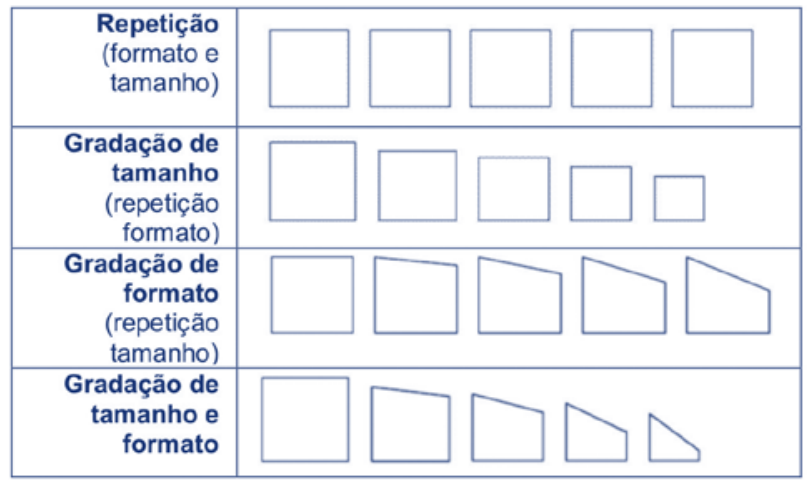

Figura 1: Operaçôes para os planos em série. Fonte - Wong (2010).

Formas mais distorcidas e complexas podem ser geradas por meio da variação de posição e/ou direção dos planos em série. A posição se refere ao espaçamento dos planos - que pode ter ou não variação (para o lado, para trás, para frente, para cima ou para baixo). A direção corresponde ao sentido de rotação - que pode ser em torno do eixo vertical ou horizontal ou em torno do seu próprio plano (que resulta num formato torcido em espiral) (Figura 2).

A partir das operaçóes definidas por Wong (2010) para a produção de formas volumétricas por meio dos planos em série, é possível identificar uma relação com o formalismo conhecido como gramática da forma (do inglês shape grammar). A gramática da forma consiste em um sistema de geraçáo de formas baseado em regras (CELANI et al., 2006). Esse sistema é desenvolvido a partir da definição de "um vocabulário básico de formas, um conjunto de regras de transformação e uma forma inicial, à qual as regras são aplicadas recursivamente, até chegar à forma desejada." (MITCHELL, 2008, p.283).

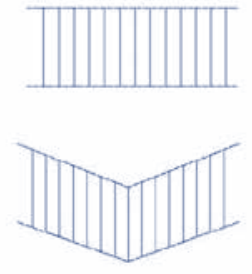

Posição (sem e com variacão)

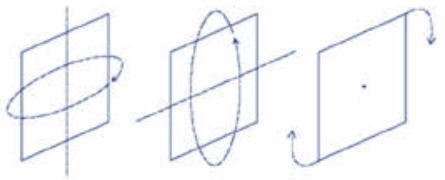

Direção
Figura 2: Elementos relacionais para as operaçóes dos planos em série. Fonte - Wong (2010).

As principais operações utilizadas nas regras de transformação de uma gramática da forma (MITCHELL, 2008; CELANI, 2003; 2006) são: a translação, a rotação, a reflexão (ou espelhamento), a transformação escalar (Figura 3), além das adiçôes, subtraçôes e substituições (Figura 4).

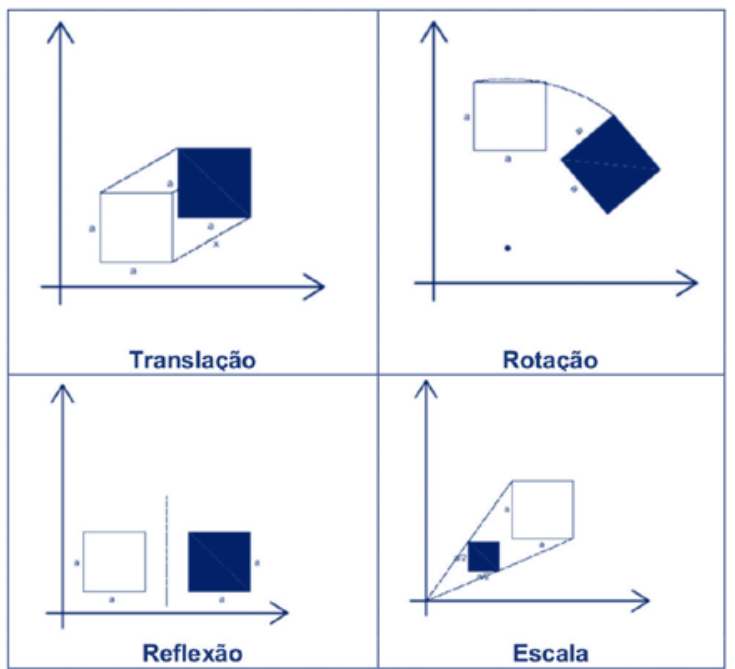

Figura 3: Transformaçôes de uma forma plana. Fonte - Mitchell (2008, p.126)

Evidencia-se, então, que de certa forma as operaçôes aplicadas nas regras de transformação da gramática da forma se assemelham às operaçóes definidas por Wong (2010) para os planos em série e que todas podem ser aplicadas a uma figura (bidimensional) primária para gerar um amplo rol de formas tridimensionais a serem fabricadas com o auxílio das cortadoras a laser (Quadro 1).

\begin{tabular}{|l|l|}
\hline Quadro l - Comparativo das operaçães de transformação \\
\hline $\begin{array}{l}\text { Planos em série } \\
\text { (WONG, 2010) }\end{array}$ & $\begin{array}{l}\text { Gramática da Forma (MIT- } \\
\text { CHELL, 2008) }\end{array}$ \\
\hline Repetição exata & Translação \\
\hline Gradação de tamanho & Escala \\
\hline Gradação de formato & Translação + subtração \\
\hline Gradação de tamanho e formato & Translação + subtração ou adição \\
\hline Posição (sem variação) & Translação \\
\hline Posição (com variação) & Translação + rotação \\
\hline Direção & Rotação \\
\hline
\end{tabular}




\section{Workshop: do plano ao volume}

O workshop estruturou-se didaticamente em cinco etapas: (1) apresentação do tema dos planos em série (com exemplos de aplicação no design e na arquitetura); (2) apresentação da gramática dos planos em série (exercício de identificaçáo das regras empregadas em alguns dos exemplos apresentados anteriormente); (3) concepçáo de peças de mobiliário utilizando os planos em série (modelagem em computador, especialmente com a utilização do software Rhinoceros); (4) prototipagem e fabricaçáo das peças com auxílio de uma cortadora a laser; (5) avaliação crítica do processo e dos resultados alcançados.

A partir da definiçáo de 3 elementos a saber: (1) cadeira, (2) luminária e (3) mesa, estes foram modelados no software Rhinoceros e após produzido o volume, foram utilizados comandos específicos (contour) para a geração dos planos em série. Em seguida, foi exportado em formato compatível $(*$.dxf) para o software da máquina de corte a laser, executadas as configuraçóes necessárias para o corte como a regulagem da potência do laser, velocidade de corte, precisáo, altura do gabarito e posterior corte das placas de MDF $6 \mathrm{~mm}$, no caso da mesa, papel Paraná 3mm, no caso da luminária e papeláo no caso da cadeira.

\section{Resultados e Discussão}

Abaixo podemos visualizar algumas das imagens do resultado final das peças.

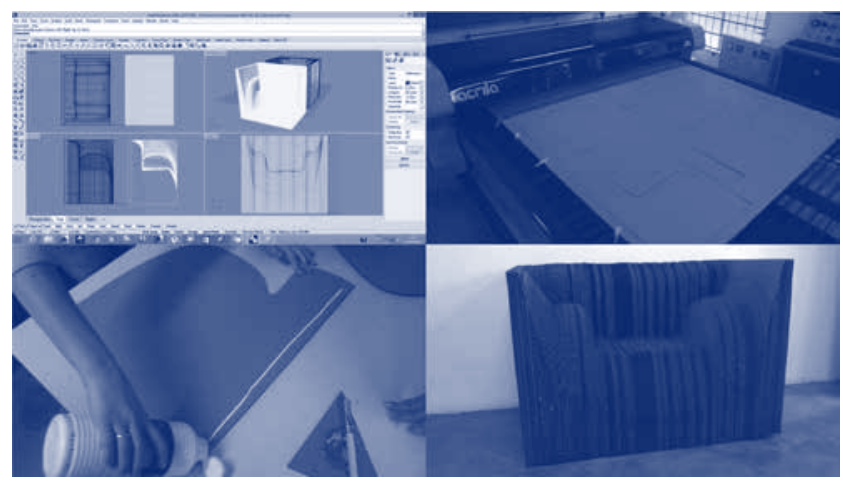

Figura 4: Processo de fabricação cadeira. Fonte - Acervo dos Autores (2014).

Em virtude da proposta de se utilizar três diferentes materiais, encontraram-se algumas dificuldades já esperadas. Toda a modelagem das peças, até o momento efetivo do corte, deu-se de maneira semelhante, embora, durante o processo de regulagem da máquina, foram necessários diferentes ajustes para cada tipo de material, a saber: (1) altura do gabarito - distância entre o material apoiado na mesa da máquina e o canhão de laser, (2) ajuste de diferentes potências do laser para cada tipo de material evitando queima do mesmo ou insuficiência de força para efetuar o corte, (3) ajuste da velocidade com que o canháo de laser passaria sobre o material em funçáo da potência escolhida anteriormente, efetivando ou não o corte desejado e por fim o ajuste da precisão do corte.

O resultado do workshop foi, como mencionado acima, a produção de três peças do mobiliário, uma luminária, uma mesa

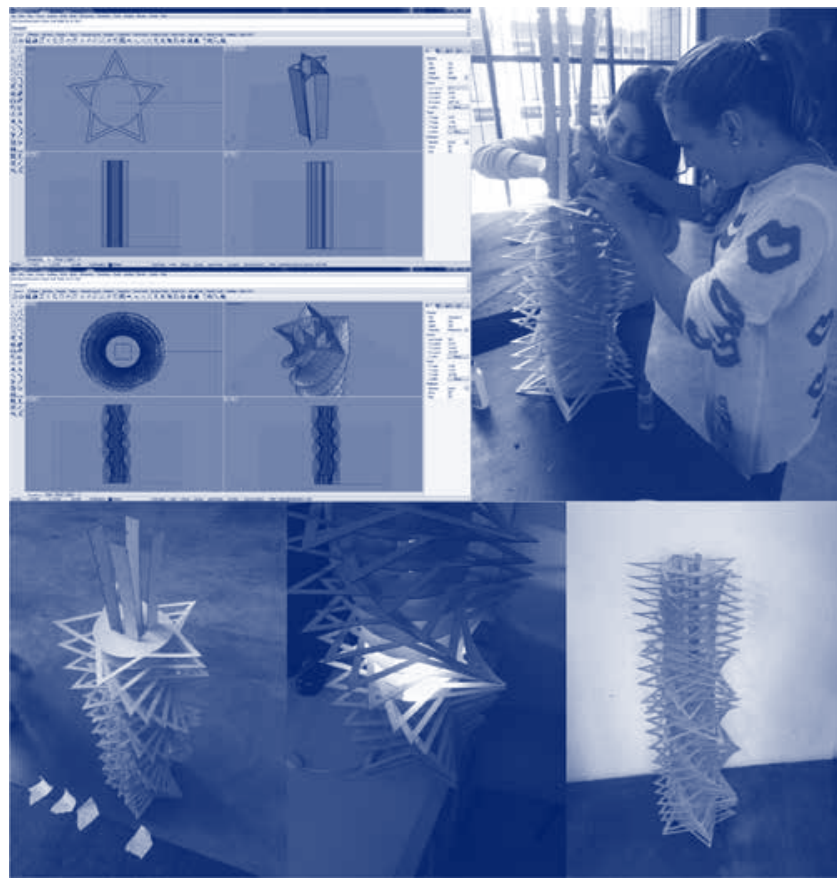

Figura 5: Processo de fabricação Luminária. Fonte-Acervo dos Autores (2014).

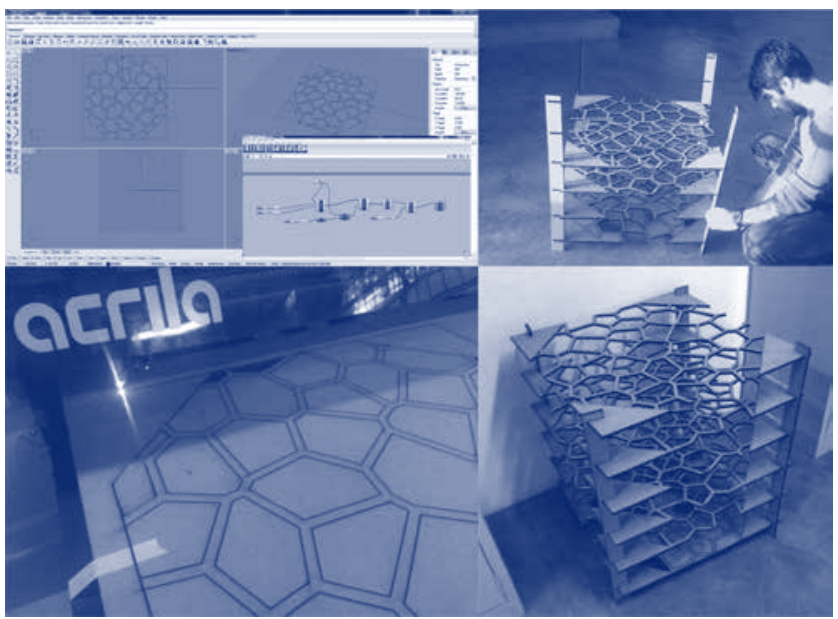

Figura 6: Processo de fabricaçáo mesa. Fonte - Acervo dos Autores (2014).

de canto e uma cadeira. O workshop mostrou-se fundamental para se verificar algumas das possibilidades de como os alunos podem se apropriar da gramática dos planos em série para concepção de formas volumétricas. Destaca-se que foram distintas as soluçóes adotadas pelos diferentes grupos, valendo-se da mesma lógica de trabalho.

Verificou-se, ao final, a validade da aplicação da gramática dos planos em série para a geraçáo de objetos tridimensionais formalmente contemporâneos, produzidos com tecnologia do corte a laser (fabricaçáo digital).

Deve-se destacar que o domínio da gramática dos planos em série possibilita o descortinamento do repertório formal possível de ser estabelecido a partir da manipulação de figuras bidimensionais 
primárias seguindo regras preestabelecidas. Assim, como já afirmado por Dunn, (2012, p.91), ratifica-se que para a concepção de formas complexas a partir da lógica dos planos em série, o computador é crucial, "já que permite aos projetistas transladar as informaçóes do projeto digital tridimensional às lâminas bidimensionais que, uma vez cortadas, proporcionam um conjunto de partes que depois se juntarão de acordo com o projeto”.

Ainda deve-se evidenciar que a gramática dos planos em série se mostra válida tanto para as análises de objetos já concebidos quanto se apresenta como um ferramental lógico passível de instrumentalizar a ação projetual que explora uma parte do universo de possibilidades das cortadoras a laser.

\section{Considerações finais}

Como reflexão, pode-se considerar o produto final como resultado de dois estudos distintos

O primeiro deles tange ao conceitual e a efetiva construção de volumes a partir de planos em série conjugados com a identificação de elementos ou transformaçóes advindas dos conceitos de gramática da forma.

O segundo refere-se à produção das peças, ao trabalho de fabricação e, de suma importância, o processo prático de utilização da tecnologia de corte a laser.

\section{Agradecimentos}

Agradecemos a todos os pesquisadores e bolsistas do Grupo de Pesquisa das Linguagens e Expressóes da Arquitetura, Urbanismo e Design (LEAUD), a Pró-Reitoria de Pesquisa da Universidade Federal de Juiz de Fora- PROPESQ/UFJF e à Fundação de Amparo à Pesquisa do Estado de Minas Gerais - FAPEMIG por viabilizar, financeiramente a apresentação deste artigo neste congresso.

\section{Referências}

DUNN, N. (2012) Proyecto y construcción digital em arquitectura. Barcelona: Blume.

CELANI, G. (2003) CAD criativo. Rio de Janeiro: Campus.

CELANI, G. et al. (2006) A gramática da forma como metodologia de análise e síntese em arquitetura. Conexão - Comunicação e Cultura, UCS, Caxias do Sul, v. 5, n. 10, jul./dez. 2006. p. 180-197.

CHING, F. D. K. (1998) Arquitetura, forma, espaço e ordem. São Paulo: Martins Fontes.

MITCHELL, W. J. (2008) A lógica da arquitetura: projeto, computação e cognição. Campinas: Editora da UNICAMP.

MUNARI, B. (1968) Design e comunicação visual. São Paulo: Martins Fontes.

PUPO, R. T. (2009) A inserção da prototipagem e fabricação digitais no processo de projeto: um novo desafio para o ensino da arquitetura. 240f. Tese (Doutorado em Engenharia Civil) - Programa de Pós-Graduação em Engenharia Civil, Faculdade de Engenharia, Arquitetura e Urbanismo, Universidade Estadual de Campinas, Campinas, SP.

STINY, G.; GIPS, J. Shape grammars and the generative specification of painting and sculpture In: IFIP CONGRESS, 7., 1972, Amsterdam. Proceedings of .... : C. V. Freimanp. 1460-1465. Disponível em: <http://www.shapegrammar.org/ifip/ifip1. html>. Acesso em: 01 set. 2014.

WONG, W. (2010). Princípios de forma e desenho. 2. ed. São Paulo: Martins Fontes. 